\title{
SPECTRAL SYNTHESIS IN SOME TOPOLOGICAL VECTOR SPACES OF FUNCTIONS
}

\author{
S. S. PLATONOV
}

\begin{abstract}
For the topological vector function spaces of exponential growth, the spectral synthesis property is proved and the spectra of invariant subspaces are described fully.
\end{abstract}

\section{$\S 1$ Introduction AND STATEMENT OF THE MAIN RESULTS}

Let $\mathcal{F}$ be an arbitrary complete locally convex space that consists of complex-valued functions (usual functions or distributions) $f(x)$ on $\mathbb{R}$ and satisfies the conditions

1) if $f(x) \in \mathcal{F}$, then $\left(\tau_{y} f\right)(x):=f(x-y) \in \mathcal{F}$ for any $y \in \mathbb{R}$;

2) for any $f \in \mathcal{F}$ the map $y \mapsto \tau_{y} f$ is a continuous map from $\mathbb{R}$ to $\mathcal{F}$.

Such a function space $\mathcal{F}$ is called a translation invariant space. As examples of translation invariant spaces we mention the space $C$ of continuous functions on $\mathbb{R}$, the space $\mathcal{E}=C^{\infty}$ of infinitely differentiable functions, the space $\mathcal{D}$ of infinitely differentiable functions on $\mathbb{R}$ with compact support, and the space $\mathcal{D}^{\prime}$ of distributions (all spaces are equipped with their usual topologies; in particular, $\mathcal{D}^{\prime}$ is endowed with the weak topology $\sigma\left(\mathcal{D}^{\prime}, \mathcal{D}\right)$ (see [1)).

A closed linear subspace $H \subseteq \mathcal{F}$ is said to be invariant if $H$ is invariant under the shifts $\tau_{y}$ for every $y \in \mathbb{R}$.

Definition 1.1. An invariant subspace $H \subseteq \mathcal{F}$ admits spectral synthesis if $H$ coincides with the closed linear span of all functions of the form

$$
x^{k} e^{i \lambda x}, \quad \lambda \in \mathbb{C}, \quad i=\sqrt{-1}, \quad k \in \mathbb{Z}_{+}=\{0,1,2, \ldots\}
$$

that belong to $H$.

For any invariant subspace $H \subseteq \mathcal{F}$, the set

$$
\Lambda=\left\{\lambda \in \mathbb{C}: e^{i \lambda x} \in H\right\}
$$

is called the spectrum of $H$. If $\lambda \in \Lambda$, we put

$$
r_{\lambda}=\sup \left\{k \in \mathbb{N}: x^{k-1} e^{i \lambda x} \in H\right\} .
$$

The quantity $r_{\lambda}$ takes values in the set $\mathbb{N} \cup\{\infty\}$. We agree that each $\lambda$ belongs to $\Lambda$ with multiplicity $r_{\lambda}$.

If an invariant subspace $H$ admits spectral synthesis, then $H$ can be recovered uniquely by its spectrum $\Lambda$; namely, $H$ coincides with the closure in $\mathcal{F}$ of the linear span of the functions

$$
x^{k} e^{i \lambda x}, \quad \lambda \in \Lambda, \quad 0 \leq k<r_{\lambda} .
$$

2010 Mathematics Subject Classification. Primary 46E10, 46F05.

Key words and phrases. Spectral synthesis, invariant subspaces, function spaces, algebras of functions, closed ideals.

(C)2011 American Mathematical Society 
We say that a translation invariant space $\mathcal{F}$ has the spectral synthesis property if any nontrivial invariant subspace $H \subset \mathcal{F}$ (i.e., not equal either to the zero subspace or to the entire space $\mathcal{F}$ ) admits spectral synthesis.

In the papers [2] and [3], L. Schwartz showed that the spaces $C, \mathcal{E}$, and $\mathcal{D}^{\prime}$ have the spectral synthesis property. Our main goal in the present paper is in constructing some classes of function spaces with the spectral synthesis property and in obtaining a description of the spectra of all possible invariant subspaces in such spaces. We note that there are other approaches to the notion of spectral synthesis (see, e.g., the survey paper 4] by Nikol'skil, the book [5], and the references therein).

For any $m \in \mathbb{Z}_{+}$and any $\nu \in \mathbb{R}$, we denote by $C_{(\nu)}^{(m)}$ the Banach space formed by all continuously differentiable functions $\varphi(x), x \in \mathbb{R}$, for which the following norm is finite:

$$
\|\varphi\|_{(\nu)}^{(m)}:=\sup _{x \in \mathbb{R}, 0 \leq \alpha \leq m} \exp (\nu|x|)\left|\partial^{\alpha} \varphi(x)\right|,
$$

where $\partial=\frac{d}{d x}$ is the operator of differentiation. For the spaces $C_{(\nu)}^{(m)}$ we have the (continuous) embeddings

$$
C_{(\nu)}^{(m)} \subseteq C_{\left(\nu^{\prime}\right)}^{\left(m^{\prime}\right)}, \quad m \geq m^{\prime}, \quad \nu \geq \nu^{\prime}
$$

The space

$$
C_{(\nu)}^{(\infty)}:=\bigcap_{m \in \mathbb{Z}_{+}} C_{(\nu)}^{(m)}
$$

is a complete locally convex space with the topology determined by the family of norms $\|\cdot\|_{(\nu)}^{(m)}$. Obviously,

$$
C_{(\nu)}^{(\infty)} \subseteq C_{\left(\nu^{\prime}\right)}^{(\infty)}, \quad \nu \geq \nu^{\prime}
$$

and this inclusion is continuous. We define the spaces

$$
\mathfrak{S}:=\bigcap_{\nu \in \mathbb{R}} C_{(\nu)}^{(\infty)}, \quad \mathfrak{D}:=\bigcup_{\nu \in \mathbb{R}} C_{(\nu)}^{(\infty)} .
$$

The space $\mathfrak{S}$ consists of infinitely differentiable functions that decay as $x \rightarrow \infty$ with all their derivatives faster than any exponential $\exp (-\nu|x|), \nu>0$, and the space $\mathfrak{D}$ consists of infinitely differential functions $\varphi$ that may grow with all their derivatives no faster than some exponential $\exp (\nu|x|), \nu=\nu(\varphi)>0$.

Let $\mathfrak{S}^{\prime}$ be the space dual to $\mathfrak{S}$, i.e., the space of all continuous linear functionals on $\mathfrak{S}$. Endowed with the weak topology $\sigma\left(\mathfrak{S}^{\prime}, \mathfrak{S}\right)$, the set $\mathfrak{S}^{\prime}$ becomes a complete locally convex space. We can say that $\mathfrak{S}^{\prime}$ is the space of distributions of exponential growth. For $f \in \mathfrak{S}^{\prime}$ and $\varphi \in \mathfrak{S}$, we denote by $\langle f, \varphi\rangle$ the value of the functional $f$ at $\varphi$. It is easy to show that $\mathfrak{D} \subseteq \mathfrak{S}^{\prime}$ (the inclusion is continuous) if we identity a function $f \in \mathfrak{D}$ with a linear functional on $\mathfrak{S}$ by the formula

$$
\langle f, \varphi\rangle:=\int_{\mathbb{R}} f(x) \varphi(x) d x,
$$

where $f \in \mathfrak{D}, \varphi \in \mathfrak{S}$. Various properties of the spaces $\mathfrak{S}, \mathfrak{S}^{\prime}$, and $\mathfrak{D}$ can be found, e.g., in 6 .

Definition 1.2. A translation invariant function space $\mathcal{F}$ is called a space of exponential growth if

$$
\mathfrak{D} \subseteq \mathcal{F} \subseteq \mathfrak{S}^{\prime}
$$

with continuous inclusions. 
We present examples of function spaces of exponential growth. Suppose $1 \leq p<\infty$ and $k \in \mathbb{R}$, and let $L_{k}^{p}$ denote the Banach space of all measurable functions $f(x)$ on $\mathbb{R}$ (viewed modulo values on sets of measure zero) for which the following norm is finite:

$$
N_{p, k}(f):=\left(\int_{\mathbb{R}}|f(x)|^{p} \exp (-k p|x|) d x\right)^{1 / p} .
$$

We endow the space

$$
L_{*}^{p}:=\bigcup_{k>0} L_{k}^{p}
$$

with the topology of the inductive limit of the Banach space $L_{k}^{p}$. It is easily seen that $L_{*}^{p}$ is a function space of exponential growth. The inclusion $L_{*}^{p} \subset \mathfrak{S}^{\prime}$ is given by formula (1.2), where $f \in L_{*}^{p}, \varphi \in \mathfrak{S}$.

As another example of a function space of exponential growth, we mention the space

$$
C_{*}^{(m)}:=\bigcup_{\nu>0} C_{(\nu)}^{(m)}, \quad m \in \mathbb{Z}_{+},
$$

equipped with the topology of the inductive limit of the Banach spaces $C_{(\nu)}^{(m)}$.

Theorem 1.1. $1^{\circ}$. Any function space of exponential growth has the spectral synthesis property.

$2^{\circ}$. Let $\mathcal{F}$ be a function space of exponential growth, and let $\Lambda$ be a collection of complex numbers, each counted with a certain multiplicity $r_{\lambda}$. The set $\Lambda$ is the spectrum of some nontrivial invariant subspace $H \subset \mathcal{F}$ if and only if the following conditions are satisfied.

(i) The set $\Lambda$ is finite or countable and has no limit points in $\mathbb{C}$, and the multiplicity $r_{\lambda}$ of each $\lambda \in \Lambda$ is finite.

(ii) For any $k>0$, consider the set

$$
\Lambda_{k}:=\{\lambda \in \Lambda:|\operatorname{Im} \lambda|<k\}
$$

and enumerate the numbers in $\Lambda_{k}$ in the order of increasing of $|\operatorname{Re} \lambda|$,

$$
0 \leq\left|\operatorname{Re} \lambda_{1}\right| \leq\left|\operatorname{Re} \lambda_{2}\right| \leq \cdots,
$$

assuming that each $\lambda \in \Lambda_{k}$ occurs $r_{\lambda}$ times in this sequence. Then

$$
\frac{\left|\operatorname{Re} \lambda_{n}\right|}{\ln n} \rightarrow \infty \text { as } n \rightarrow \infty
$$

unless the set $\Lambda_{k}$ is finite.

Theorem 1.1 gives a full description of the invariant subspaces in any function space of exponential growth. The proof of this theorem is our main goal in the present paper.

A few words about the origin of the problem are in order. Let $L_{* o}^{2}$ denote the subspace of odd functions in the space $L_{*}^{2}$. In [7, Rashevskiu described all closed linear subspaces in $L_{* o}^{2}$ that are invariant with respect to the transformations

$$
f(x) \mapsto \frac{1}{2}(f(x+y)+f(x-y)), \quad y \in \mathbb{R} .
$$

The description obtained in [7] was stated in terms close to those in Theorem 1.1.

The paper [8] by Gilbert is also related to the description of invariant subspaces in spaces of exponential growth. There, convolution topological algebras $\mathcal{A}$ were considered under the following assumptions:

(i) $\mathfrak{S} \subseteq \mathcal{A} \subseteq \mathfrak{D}^{\prime}\left(\mathfrak{D}^{\prime}\right.$ is the space dual to $\mathfrak{D}$; the inclusions are continuous);

(ii) the convolution $(f, g) \mapsto f * g$ is a separately continuous map from $\mathcal{A} \times \mathcal{A}$ into $\mathcal{A}$; 
(iii) $\mathcal{A}$ contains an approximate identity $\left\{\varphi_{n}\right\}$ formed by functions $\varphi_{n} \in \mathfrak{S}$. Such function algebras will be called Gilbert algebras.

Let

$$
\Phi: f(x) \mapsto \widehat{f}(z)=\int_{\mathbb{R}} f(x) e^{i x z} d x, \quad z \in \mathbb{C},
$$

be the Fourier transformation. For any convolution Gilbert algebra $\mathcal{A}$, its image $\Phi(\mathcal{A})=$ $\widehat{\mathcal{A}}$ is a topological algebra of entire functions with the pointwise multiplication. If $I$ is a closed ideal in $\mathcal{A}$, then $\widehat{I}:=\Phi(I)$ is a closed ideal in $\widehat{\mathcal{A}}$. The set $\Lambda$ of common zeros of all functions in $\widehat{I}$ (counting multiplicities) is called the cospectrum of the ideal $I$. The ideal $\widehat{I}$ is said to be localizable in $\widehat{\mathcal{A}}$ if it coincides with the set of all functions in $\widehat{\mathcal{A}}$ that vanish at all points of $\mathcal{A}$ (with the corresponding multiplicities). In 8 it was proved that, for any closed ideal $I$ in a Gilbert algebra $\mathcal{A}$, the corresponding ideal $\widehat{I}$ is localizable in $\widehat{\mathcal{A}}$ and, thus, is determined uniquely by its cospectrum.

The main part of the proof of Theorem 1.1 is the verification of it in the case where $\mathcal{F}$ coincides with the space $L_{*}^{2}$. Then, the dual space $\left(L_{*}^{2}\right)^{\prime}=M_{*}^{2}$ is a convolution topological Gilbert algebra (it was denoted by $L_{\omega}^{2}$ in $\left[8\right.$ ). The invariant subspaces in $L_{*}^{2}$ are in one-to-one correspondence with the closed ideals in $M_{*}^{2}$ : each invariant subspace $H \subseteq L_{*}^{2}$ gives rise to the closed ideal

$$
H^{\perp}=\left\{f \in M_{*}^{2}:\langle f, g\rangle=0, g \in H\right\} \subset M_{*}^{2} .
$$

Applying the Fourier transformation, we reduce the description of closed ideals in the convolution algebra $M_{*}^{2}$ to the description of closed ideals in the topological algebra $\mathcal{A}_{*}^{2}:=\Phi\left(M_{*}^{2}\right)$ of entire functions. Under this reduction, an invariant subspace $H \subseteq L_{*}^{2}$ admits spectral synthesis if and only if the ideal $\Phi\left(H^{\perp}\right)$ is localizable in $\mathcal{A}_{*}^{2}$, and the spectrum of $H$ coincides with the cospectrum of the ideal $H^{\perp}$, i.e., with the set of common zeros of the ideal $\Phi\left(H^{\perp}\right)$.

Since $M_{*}^{2}$ is a Gilbert algebra, all closed ideals in $\mathcal{A}_{*}^{2}$ are localizable; thus, the spectral synthesis property for $L_{*}^{2}$ is implied by the results of $[8$, but without any description of the invariant subspaces' spectra. In the present paper, we present a new proof of the spectral synthesis property in $L_{*}^{2}$, which also allowed us to describe the spectra of the invariant subspaces. We note that some elements of the proof of Theorem 1.1 are parallel to the corresponding points of the proofs in [8, but the most essential parts differ: the proof in [8] is based on the Beurling-Lax theorem pertaining to invariant subspaces in the Hardy space $H^{2}(\mathbb{R})$, while in the present paper we employ methods close to those in Rashevskiıı's paper [7].

Also, in $\S 4$ we present two other classes of translation invariant spaces, for which we prove the spectral synthesis property and describe the spectra of invariant subspaces.

The author thanks the referees for valuable remarks.

\section{$\S 2$. REDUCTION OF THE SPECTRAL SYNTHESIS PROBLEM IN SPACES OF EXPONENTIAL GROWTH TO THAT IN THE SPACE $L_{*}^{2}$}

Let $\mathcal{F}$ be an arbitrary translation invariant function space of exponential growth, and let $f(x) \in \mathcal{F}, \varphi(x) \in \mathcal{D}$. The convolution $f * \varphi$ can be defined by the formula

$$
f * \varphi:=\int_{\mathbb{R}}\left(\tau_{y} f\right) \varphi(y) d y
$$

where $\left(\tau_{y} f\right)(x)=f(x-y)$ is the shift operator. Since the map $y \mapsto \tau_{y} f$ is a continuous vector-valued function on $\mathbb{R}$ with values in the complete locally convex space $\mathcal{F}$, the integral on the right in (2.1) can be thought of as a Riemann integral of a vector-valued function. If $H$ is an invariant subspace in $\mathcal{F}$ and $f \in H$, then (2.1) shows that $f * \varphi \in H$. 
On the other hand, since $\mathcal{F} \subseteq \mathfrak{S}^{\prime} \subset \mathcal{D}^{\prime}$, the convolution $f * \varphi$ can be viewed as the convolution of a test function and a distribution (see, e.g., [9, Chapter II, §12]), i.e.,

$$
(f * \varphi)(x):=\left\langle f, \tau_{-x} \varphi\right\rangle .
$$

Lemma 2.1. If $f \in \mathfrak{S}^{\prime}$ and $\varphi \in \mathcal{D}$, then $f * \varphi \in \mathfrak{D}$ and the map $f \mapsto f * \varphi$ from $\mathfrak{S}^{\prime}$ to $\mathfrak{D}$ is continuous.

Proof. It is well known (see 9, Chapter II, §12]) that, if $f \in \mathcal{D}^{\prime}$ and $\varphi \in \mathcal{D}$, then the function $f * \varphi$ is infinitely differentiable, and

$$
\partial^{k}(f * \varphi)(x)=\left(f * \partial^{k} \varphi\right)(x), \quad k \in \mathbb{Z}_{+} .
$$

It is also known (see [6. Chapter III, $\S 1]$ ) that the topological vector space $\mathfrak{S}^{\prime}$ can be identified with the inductive limit of the Banach spaces dual to the spaces $C_{(\nu)}^{(m)}, m \in \mathbb{Z}_{+}$, $\nu>0$ :

$$
\mathfrak{S}^{\prime}=\bigcup_{m, \nu}\left(C_{(\nu)}^{(m)}\right)^{\prime}
$$

i.e., any functional $f \in \mathfrak{S}^{\prime}$ extends up to a continuous linear functional on a Banach space $C_{(\nu)}^{(m)}$ with some $m \in \mathbb{Z}_{+}, \nu>0$.

Let $A$ be the norm of $f$ viewed as a functional of the Banach space $C_{(\nu)}^{(m)}$, i.e.,

$$
A=\sup \left\{|\langle f, \psi\rangle|: \psi \in C_{(\nu)}^{(m)},\|\psi\|_{(\nu)}^{(m)} \leq 1\right\} .
$$

Then

$$
\left|\partial^{k}(f * \varphi)(x)\right|=\left|\left\langle f, \partial^{k}\left(\tau_{-x} \varphi\right)\right\rangle\right| \leq A\left\|\partial^{k}\left(\tau_{-x} \varphi\right)\right\|_{(\nu)}^{(m)} .
$$

Observe that

$$
\begin{aligned}
\left\|\partial^{k}\left(\tau_{-x} \varphi\right)\right\|_{(\nu)}^{(m)} & =\sup _{y \in \mathbb{R}, 0 \leq \alpha \leq m} \exp (\nu|y|)\left|\partial^{k+\alpha} \varphi(x+y)\right| \\
& =\sup _{\xi \in \mathbb{R}, 0 \leq \alpha \leq m} \exp (\nu|\xi-x|)\left|\partial^{k+\alpha} \varphi(\xi)\right| \leq \exp (\nu|x|)\|\varphi\|_{(\nu)}^{(m+k)} .
\end{aligned}
$$

Inequalities (2.3) and (2.4) imply that

$$
\left|\partial^{k}(f * \varphi)(x)\right| \leq A \exp (\nu|x|)\|\varphi\|_{(\nu)}^{(k+m)},
$$

whence we see that $f * \varphi \in C_{(\nu)}^{(\infty)} \subset \mathfrak{D}$.

Estimate (2.5) also shows that the map $f \mapsto f * \varphi$ from $\left(C_{(\nu)}^{(m)}\right)^{\prime}$ to $\mathfrak{D}$ is continuous; since $\mathfrak{S}^{\prime}$ is the inductive limit of the spaces $\left(C_{(\nu)}^{(m)}\right)^{\prime}$, this map is continuous as a map from $\mathfrak{S}^{\prime}$ to $\mathfrak{D}$.

By an approximate identity we shall mean a sequence of functions $\varphi_{n}(x) \in \mathcal{D}, n=$ $1,2, \ldots$, such that 1) $\left.\varphi_{n}(x) \geq 0 ; 2\right) \operatorname{supp} \varphi_{n} \subseteq[-1 / n, 1 / n]$; 3) $\int_{\mathbb{R}} \varphi_{n}(x) d x=1(\operatorname{supp} \varphi$ is the support of $\varphi$ ). If $\mathcal{F}$ is an arbitrary translation invariant function space, then for any $f \in \mathcal{F}$ the sequence $f * \varphi_{n}$ converges to $f$ in the topology of $\mathcal{F}$. Indeed, let $V$ be any closed convex neighborhood of the point $f$ in $\mathcal{F}$. Since the map $y \mapsto \tau_{y} f=f(x-y)$ is continuous from $\mathbb{R}$ to $\mathcal{F}$ (this follows from the definition of a translation invariant space), we can find $\varepsilon>0$ such that $\tau_{y} f \in V$ for all $y$ with $|y| \leq \varepsilon$. Then the properties of an approximate identity show that

$$
f * \varphi_{n}=\int_{\mathbb{R}}\left(\tau_{y} f\right) \varphi_{n}(y) d y \in V
$$

for $n>1 / \varepsilon$. 
We denote by $s(\mathcal{F})$ the collection of all invariant subspaces of $\mathcal{F}$. Suppose that $\mathcal{F}$ has exponential growth; then $\mathfrak{D} \subseteq \mathcal{F}$. We define two maps $\rho: s(\mathcal{F}) \mapsto s(\mathfrak{D})$ and $\widetilde{\rho}: s(\mathfrak{D}) \mapsto s(\mathcal{F})$ by the formulas

$$
\begin{aligned}
\rho(H) & :=H \cap \mathfrak{D}, \quad H \in s(\mathcal{F}), \\
\widetilde{\rho}\left(H_{0}\right) & :=\left[H_{0}\right], \quad H_{0} \in s(\mathfrak{D}),
\end{aligned}
$$

where $\left[H_{0}\right]$ is the closure of the set $H_{0}$ in $\mathcal{F}$ (if we need to indicate the space where closure is taken, we shall write $\left[H_{0}\right]_{\mathcal{F}}$ in place of $\left.\left[H_{0}\right]\right)$.

Theorem 2.1. For any function space $\mathcal{F}$ of exponential growth, the map $\rho$ is a bijection from $s(\mathcal{F})$ onto $s(\mathfrak{D})$. The inverse map $\rho^{-1}$ coincides with $\widetilde{\rho}$.

Proof. Let $H$ be an arbitrary invariant subspace in $\mathcal{F}$, and let $H_{0}=H \cap \mathfrak{D}$. If $f \in H$ and $\left\{\varphi_{n}\right\}_{n \in \mathbb{N}}$ is an approximate identity, then $f * \varphi_{n} \in H \cap \mathfrak{D}=H_{0}$ by Lemma 2.1. Since $f * \varphi_{n} \rightarrow f$ in $\mathcal{F}$ as $n \rightarrow \infty$, we have $f \in\left[H_{0}\right]$. Consequently, $\left[H_{0}\right]=H$, i.e., $\widetilde{\rho} \circ \rho=I d_{s(\mathcal{F})}\left(I d_{A}\right.$ denotes the identical map of the set $A$ onto itself $)$.

Now, let $H_{0}$ be an arbitrary invariant subspace in $\mathfrak{D}$ and let $H=\left[H_{0}\right]$. If $f \in H \cap \mathfrak{D}$, then there is a net $\left\{h_{\alpha}\right\}_{\alpha \in X}\left(X\right.$ is some directed set) such that $h_{\alpha} \in H_{0}$ and $h_{\alpha} \stackrel{\alpha \in X}{\longrightarrow} f$ in $\mathcal{F}$. Let $\varphi_{n}$ be an approximate identity; Lemma 2.1 implies that $h_{\alpha} * \varphi_{n} \stackrel{\alpha \in X}{\longrightarrow} f * \varphi_{n}$ in $\mathfrak{D}$. Since $h_{\alpha} * \varphi_{n} \in H_{0}$ and the subspace $H_{0}$ is closed in $\mathfrak{D}$, we have $f * \varphi_{n} \in H_{0}$. Since $f \in \mathfrak{D}$, it follows that $f * \varphi_{n} \rightarrow f$ in $\mathfrak{D}$ as $n \rightarrow \infty$, whence $f \in H_{0}$. Thus, $H \cap \mathfrak{D}=H_{0}$, i.e., $\rho \circ \widetilde{\rho}=\operatorname{Id}_{s(\mathfrak{D})}$.

Remark 2.1. The above proof of Theorem 2.1 is similar to that of Theorem 3 in 8 .

Theorem 2.2. If a function space $\mathcal{F}$ of exponential growth has the spectral synthesis property, then all such spaces have this property.

Proof. Suppose $\mathcal{F}$ is a space of exponential growth and with the spectral synthesis property; first we check that $\mathfrak{D}$ possesses this property.

Let $H_{0}$ be an arbitrary invariant subspace in $\mathfrak{D}$, and let $H=\widetilde{\rho}\left(H_{0}\right)$ be the closure of $H_{0}$ in $\mathcal{F}$. We denote by $E$ the linear hull of all functions of the form $x^{k} e^{i \lambda x}(\lambda \in \mathbb{C}$, $k \in \mathbb{Z}_{+}$) that belong to $H$. Since $H$ admits spectral synthesis, we have $[E]_{\mathcal{F}}=H$. Observe that $E \subset \mathfrak{D}$; therefore, Theorem 2.1 implies that $E \subset H \cap \mathfrak{D}=H_{0}$. Let $H_{0}^{\prime}$ denote the closure of $E$ in $\mathfrak{D}$. Then $H_{0}^{\prime}$ is an invariant subspace in $\mathfrak{D}$ and $\widetilde{\rho}\left(H_{0}^{\prime}\right)$ coincides with $H$. Since $\widetilde{\rho}$ is a bijection of $s(\mathfrak{D})$ onto $s(\mathcal{F})$, we see that $H_{0}^{\prime}=H_{0}$; i.e., $H_{0}$ admits spectral synthesis.

Now we prove that the spectral synthesis property occurs in any function space $\mathcal{F}^{\prime}$ of exponential growth. Let $H$ be an invariant subspace in $\mathcal{F}^{\prime}$. Put $H_{0}=H \cap \mathfrak{D} \in s(\mathfrak{D})$; then

$$
\left[H_{0}\right]_{\mathcal{F}^{\prime}}=H
$$

by Theorem 2.1. Let $E$ denote the linear hull of all functions $x^{k} e^{i \lambda x}\left(\lambda \in \mathbb{C}, k \in \mathbb{Z}_{+}\right)$ that belong to $H_{0}$. Since the space $\mathfrak{D}$ has the spectral synthesis property, it follows that $[E]_{\mathfrak{D}}=H_{0}$, and (2.6) implies $[E]_{\mathcal{F}^{\prime}}=\left[H_{0}\right]_{\mathcal{F}^{\prime}}=H$; i.e., $H$ admits spectral synthesis.

Remark 2.2. The proof of Theorem 2.2 shows that the description of the spectra of all invariant subspaces does not depend on the choice of a function space of exponential growth. 


\section{§3. Proof of Theorem 1.1}

Theorem 2.2 implies that it suffices to prove Theorem 1.1 in the special case where $\mathcal{F}=L_{*}^{2}$ (for the definition of the Banach spaces $L_{k}^{p}, k \in \mathbb{R}$, and of the locally convex space $L_{*}^{p}$, see $\S 1 ; N_{p, k}$ is the norm in $L_{k}^{p}$ ).

For $k>0$ we denote the Banach space $L_{-k}^{p}$ by $M_{k}^{p}$; let $\nu_{p, k}(f):=N_{p,(-k)}(f)$ be the norm in $M_{k}^{p}$. We define the space

$$
M_{*}^{p}:=\bigcap_{k>0} M_{k}^{p}
$$

The set $M_{*}^{p}$ is a locally convex space, being endowed with the topology of the projective limit of the Banach spaces $M_{k}^{p}$, i.e., the topology given by the family of norms $\nu_{p, k}, k>0$. It is easily seen that the locally convex space $M_{*}^{p}, 1 \leq p<\infty$, is a topological algebra with respect to convolution, and that a closed linear subspace $P \subset M_{*}^{p}$ is invariant if and only if $P$ is a closed ideal of the algebra $M_{*}^{p}$.

It can readily be proved that the topological vector spaces $L_{*}^{2}$ and $M_{*}^{2}$ are dual to each other; i.e., the space dual to $L_{*}^{2}$ is isomorphic to $M_{*}^{2}$ and vice versa. The duality between $L_{*}^{2}$ and $M_{*}^{2}$ is given by

$$
\langle f, g\rangle=\int_{\mathbb{R}} f(x) g(x) d x, \quad f \in L_{*}^{2}, g \in M_{*}^{2} .
$$

The Hahn-Banach theorem shows that there is a one-to-one correspondence between the invariant subspaces in $L_{*}^{2}$ and in $M_{*}^{2}$ : to an invariant subspace $H \subset L_{*}^{2}$, we can assign the invariant subspace

$$
P=H^{\perp}=\left\{g \in M_{*}^{2}:\langle f, g\rangle=0, f \in H\right\} \subset M_{*}^{2} .
$$

Let $\mathcal{A}_{*}^{2}$ denote the set of all entire functions $F(z), z=u+i v \in \mathbb{C}$, that satisfy the condition

$$
\forall k>0 \quad \mathfrak{n}_{k}(F):=\sup _{|v| \leq k}\left(\int_{\mathbb{R}}|F(u+i v)|^{2} d u\right)^{1 / 2}<\infty \text { for all } k>0 .
$$

The set $\mathcal{A}_{*}^{2}$ is a locally convex space with the topology given by the family of seminorms (even norms) $\mathfrak{n}_{k}, k>0$.

Let $\Phi$ be the Fourier transformation (see (1.6)).

Lemma 3.1. The Fourier transformation $\Phi$ establishes an isomorphism of the topological vector spaces $M_{*}^{2}$ and $\mathcal{A}_{*}^{2}$.

Proof. Let $f(x) \in M_{*}^{2}$, and let

$$
F(z)=\Phi(f)(z)=\int_{\mathbb{R}} f(x) e^{-v x} e^{i u x} d x, \quad z=u+i v .
$$

The Parseval identity for the Fourier transformation yields

$$
2 \pi \int_{\mathbb{R}}|f(x)|^{2} e^{-2 v x} d x=\int_{\mathbb{R}}|F(u+i v)|^{2} d u .
$$

We check the relations

$$
\pi \nu_{2, k}^{2}(f) \leq \mathfrak{n}_{k}^{2}(F) \leq 2 \pi \nu_{2, k}^{2}(f), \quad k>0 .
$$

The right inequality in (3.3) is implied by (3.2) and the fact that

$$
e^{-2 v x} \leq e^{2 k|x|}
$$

if $|v| \leq k$ and $x \in \mathbb{R}$. Using (3.2) with $v=k$ and $v=-k$, together with the estimate

$$
e^{2 k x}+e^{-2 k x} \geq e^{2 k|x|}
$$


we obtain

$$
\begin{aligned}
2 \mathfrak{n}_{k}^{2}(F) & \geq \int_{\mathbb{R}}\left(|F(u+i k)|^{2}+|F(u-i k)|^{2}\right) d u=2 \pi \int_{\mathbb{R}}|f(x)|^{2}\left(e^{2 k x}+e^{-2 k x}\right) d x \\
& \geq 2 \pi \int_{\mathbb{R}}|f(x)|^{2} e^{2 k|x|} d x=2 \pi \nu_{2, k}^{2}(f),
\end{aligned}
$$

which proves the left inequality in (3.3). Inequalities (3.3) show that $F \in \mathcal{A}_{*}^{2}$ and that $\Phi$ is a continuous and injective map from $M_{*}^{2}$ to $\mathcal{A}_{*}^{2}$.

Now, let $F(z)$ be an arbitrary function of class $\mathcal{A}_{*}^{2}$. For each fixed $v$, the function $F(u+i v)$ belongs to $L^{2}(\mathbb{R})$. Denote by

$$
f_{v}(x)=\frac{1}{2 \pi} \int_{\mathbb{R}} F(u+i v) e^{-i u x} d x
$$

the inverse Fourier transform of the function $F(u+i v)$. The function $f_{0}(x)$ will be denoted by $f(x)$. It is well known (see [10, Theorem 97]) that

$$
f_{v}(x)=f(x) e^{-v x} .
$$

Since the Fourier transform of $f_{v}(x)$ coincides with $F(u+i v)$, formula (3.4) shows that $\Phi(f)=F$. Using the Parseval identity for the inverse Fourier transformation, we get formula (3.2), which implies (3.3). From inequalities (3.3) it follows that $f \in M_{*}^{2}$ and that the map $\Phi^{-1}: F \mapsto f$ from $\mathcal{A}_{*}^{2}$ to $M_{*}^{2}$ is continuous.

Since $M_{*}^{2}$ is a convolution algebra, and the Fourier transformation takes convolution to product, the space $\mathcal{A}_{*}^{2}=\Phi\left(M_{*}^{2}\right)$ is an algebra relative to the pointwise multiplication of functions.

Let $H$ be an invariant subspace in $L_{*}^{2}, H^{\perp}$ the orthogonal complement of $H$ in $M_{*}^{2}$, and $I=\Phi\left(H^{\perp}\right)$. Then $I$ is a closed ideal in the algebra $\mathcal{A}_{2}^{*}$. We shall say that the ideal $I$ corresponds to the subspace $H$. The correspondence $H \mapsto I$ is a bijection of the set of all invariant subspaces in $L_{*}^{2}$ onto the set of all closed ideals in $\mathcal{A}_{*}^{2}$.

Let $I$ be a nonzero closed ideal in $\mathcal{A}_{*}^{2}$, and let $\lambda \in \mathbb{C}, r \in \mathbb{Z}_{+}$. We say that $\lambda$ is a zero of $I$ of multiplicity $r$ if

$$
F(\lambda)=F^{\prime}(\lambda)=\cdots=F^{(r-1)}(\lambda)=0 \quad \text { for all } F \in I
$$

and there exists $G \in I$ such that $G^{(r)}(\lambda) \neq 0$. The multiplicity of $\lambda$ will be denoted by $r_{\lambda}$. Let $\mathfrak{N}_{I}$ be the set of all zeros of the ideal $I$ (each zero $\lambda$ is counted with its multiplicity $r_{\lambda}$ ).

Lemma 3.2. Let $H$ be an invariant subspace in $L_{*}^{2}$, let $\Lambda_{H}$ be its spectrum, and let I be the closed ideal in $\mathcal{A}_{*}^{2}$ that corresponds to $H$. Then $\Lambda_{H}=\mathfrak{N}_{I}$.

Proof. Let $\lambda$ belong to $\Lambda_{H}$ with multiplicity $r$; then the functions $e^{i \lambda x}, x e^{i \lambda x}, \ldots$, $x^{r-1} e^{i \lambda x}$ belong to $H$. Take $f(x) \in H^{\perp}$ and put $F(z)=\Phi(f)$. Observe that

$$
\left\langle x^{k} e^{i \lambda x}, f(x)\right\rangle=(-i)^{k} F^{(k)}(\lambda), \quad k \in \mathbb{Z}_{+} .
$$

Since the functions $x^{k} e^{i \lambda x}$ are orthogonal to $f$ for $0 \leq k \leq r-1$, we have

$$
F(\lambda)=F^{\prime}(\lambda)=\cdots=F^{(r-1)}(\lambda)=0 \text { for all } F \in I,
$$

whence $\Lambda_{H} \subset \mathfrak{N}_{I}$.

Conversely, if (3.7) is true, then (3.6) shows that $x^{k} e^{i \lambda x} \in H$ for $k \leq r-1$, implying $\mathfrak{N}_{I} \subset \Lambda_{H}$.

We shall say that a closed ideal $I \subset \mathcal{A}_{*}^{2}$ is localizable if any function $F \in \mathcal{F}_{*}^{2}$ that has a zero of multiplicity at least $r_{\lambda}$ at any point $\lambda \in \mathfrak{N}_{I}$ belongs to $I$. Any localizable ideal is determined uniquely by the set of its zeros. 
Lemma 3.3. An invariant subspace $H \subset L_{*}^{2}$ admits spectral synthesis if and only if the ideal $I \subset \mathcal{A}_{*}^{2}$ that corresponds to $H$ is localizable.

Proof. $1^{\circ}$. Suppose $H$ admits spectral synthesis; we prove that the ideal $I=\Phi\left(H^{\perp}\right)$ is localizable.

Let $F \in \mathcal{A}_{*}^{2}$ be a function such that, for any $\lambda \in \mathfrak{N}_{I}=\Lambda_{H}$, at the point $\lambda$ this function has a zero of multiplicity at least $r_{\lambda}$, and let $f=\Phi^{-1}(F) \in M_{*}^{2}$. Relation (3.6) implies that $\left\langle x^{k} e^{i \lambda x}, f\right\rangle=0$ for $\lambda \in \Lambda_{H}$ and $k \leq r_{\lambda}-1$, and since $H$ admits spectral synthesis, it follows that $f \in H^{\perp}$. Consequently, $F=\Phi(f) \in I$.

$2^{\circ}$. Conversely, let $I$ be localizable. We denote by $H_{1}$ the closure in $L_{*}^{2}$ of the linear hull of all functions $x^{k} e^{i \lambda x}$ with $\lambda \in \Lambda_{H}=\mathfrak{N}_{I}, 0 \leq k \leq r_{\lambda}-1$. Obviously, $H_{1}$ is an invariant subspace in $L_{*}^{2}$. Let $I_{1}=\Phi\left(H_{1}^{\perp}\right)$. Using (3.6), we see that any function $F \in I_{1}$ has a zero of multiplicity at least $r_{\lambda}$ at any point $\lambda \in \mathfrak{N}_{I}$, so that $F \in I$. Consequently, $I_{1}=I$, whence $H_{1}=H$, i.e., $H$ admits spectral synthesis.

Lemma 3.3 shows that for the proof of Theorem 1.1 it suffices to prove the following.

Theorem 3.1. 1) Any nontrivial closed ideal $I \subset \mathcal{A}_{*}^{2}$ is localizable.

2) A set $\Lambda$ of complex numbers (each $\lambda \in \Lambda$ is counted with its multiplicity $r_{\lambda}$ ) coincides with the set of zeros of some nontrivial closed ideal of the algebra $\mathcal{A}_{*}^{2}$ if and only if conditions $1^{\circ}$ and $2^{\circ}$ of Theorem 1.1 are fulfilled.

Before proving Theorem 3.1, we verify several auxiliary statements. In what follows, $z=u+i v \in \mathbb{C}, k>0$. Let $\Pi_{k}:=\{z:|v|<k\}$. We denote by $\mathcal{A}_{k}^{2}$ the set of all functions $F(z)$ analytic in the strip $\Pi$ and such that

$$
\mathfrak{n}_{k}(F)=\sup _{|v|<k}\left(\int_{\mathbb{R}}|F(u+i v)|^{2} d u\right)^{1 / 2}<\infty .
$$

The set $\mathcal{A}_{k}^{2}$ is a Banach space with the norm $\mathfrak{n}_{k}$.

Lemma 3.4. Let $\Lambda=\left\{\lambda_{n}=u_{n}+i v_{n}\right\}$ be a finite or countable collection of complex numbers lying in the strip $\Pi_{\pi / 4}$; each $\lambda \in \Lambda$ is counted with a finite multiplicity $r_{\lambda}$.

The set $\Lambda$ coincides with the set of zeros of some nonzero bounded function $F(z) \in$ $\mathcal{A}_{\pi / 4}^{2}$ (each $\lambda \in \Lambda$ must be a zero of $F$ with multiplicity $r_{\lambda}$ ) if and only if

$$
\sum_{n} e^{-2\left|u_{n}\right|} \cos 2 v_{n}<\infty .
$$

Proof. 1) The "only if" part. The map $\alpha: z \mapsto w=\tan (i z)$ is a conformal one-toone mapping of the strip $\Pi_{\pi / 4}$ onto the unit disk $D=\{w \in \mathbb{C}:|w|<1\}$. Let $\Lambda=$ $\left\{\lambda_{n}=u_{n}+i v_{n}\right\}$ be the set of zeros of a bounded function $F \in \mathcal{A}_{\pi / 4}^{2}$. Then the function $G(w):=F\left(\alpha^{-1}(w)\right)$ is analytic and bounded in $D$, and the numbers $\gamma_{n}=\tan \left(i \lambda_{n}\right)$ are the zeros of $G$. It is well known that a finite or countable collection of points $\gamma_{n} \in D$ coincides with the set of zeros of some nonzero bounded function analytic in $D$ if and only if the Blaschke condition is satisfied:

$$
\sum_{n}\left(1-\left|\gamma_{n}\right|\right)<\infty
$$

Obviously, (3.10) is equivalent to

$$
\sum_{n}\left(1-\left|\gamma_{n}\right|^{2}\right)<\infty
$$

If $\lambda=u+i v, \gamma=\tan (i \lambda)$, then

$$
1-|\gamma|^{2}=\frac{\left(1-\tanh ^{2} u\right)\left(1-\tan ^{2} v\right)}{1+\tanh ^{2} u \tan ^{2} v}=\frac{\cos 2 v(\cosh u)^{-2}}{\cos ^{2} v\left(1+\tanh ^{2} u \tan ^{2} v\right)} .
$$


Using the estimates

$$
\frac{1}{2} e^{|u|} \leq \cosh u \leq e^{|u|}
$$

and the fact that $|v|<\pi / 4$, from (3.12) we obtain

$$
\frac{1}{2} e^{-2|u|} \cos 2 v \leq 1-|\gamma|^{2} \leq 8 e^{-2|u|} \cos 2 v,
$$

which shows that (3.11) is equivalent to (3.9).

2) The "if" part. Suppose (3.9) is fulfilled; then the numbers $\gamma_{n}=\tan \left(i \lambda_{n}\right)$ satisfy (3.10). Therefore, we can find a nonzero function $G(w)$, analytic and bounded in the disk $D$, for which the set $\left\{\gamma_{n}\right\}$ is the set of zeros. The function $H(z)=G(\tan (i z))$ will be analytic and bounded in the strip $\Pi_{\pi / 4}$, the set $\Lambda=\left\{\lambda_{n}\right\}$ will coincide with the set of zeros of $H$, but, in general, $H(z)$ may fail to belong to the class $\mathcal{A}_{\pi / 2}^{2}$. If we take $F(z)=e^{-z^{2}} H(z)$, we obtain a bounded function of class $\mathcal{A}_{\pi / 4}^{2}$ with the same set of zeros.

Corollary 3.1. A finite or countable collection $\Lambda=\left\{\lambda_{n}=u_{n}+i v_{n}\right\}$ lying in a strip $\Pi_{k}$ is the set of zeros of some nonzero bounded function of class $\mathcal{A}_{k}^{2}$ if and only if

$$
\sum_{n} e^{-\frac{\pi}{2 k}\left|u_{n}\right|} \cos \frac{\pi}{2 k} v_{n}<\infty .
$$

Proof. The transformation $z \mapsto \frac{\pi z}{4 k}$ takes $\Pi_{k}$ onto $\Pi_{\pi / 4}$, and (3.14) will be reshaped to (3.9).

Remark 3.1. Let $F(z)$ be a nonzero bounded function of class $\mathcal{A}_{k}^{2}$. If $\widetilde{\Lambda}=\left\{\lambda_{n}=\right.$ $\left.u_{n}+i v_{n}\right\}$ is a collection of (not necessarily all) zeros of $F$, and each $\lambda \in \widetilde{\Lambda}$ is counted with some multiplicity not exceeding the multiplicity of $\lambda$ as a zero of $F$, then $\widetilde{\Lambda}$ satisfies condition (3.14).

In the next lemma we collect some properties of functions of class $\mathcal{A}_{k}^{2}$ needed in what follows. If $F(z) \in \mathcal{A}_{k}^{2}, z=u+i v$, then, for every fixed $v \in(-k, k)$, the function $F(u+i v)$ belongs to $L^{2}(\mathbb{R})$ as a function of $u$.

Lemma 3.5. $1^{\circ}$. If $F(z) \in \mathcal{A}_{k}^{2}$, then $F(u+i v) \rightarrow 0$ as $u \rightarrow \infty$ uniformly in every strip $|v| \leq k-\varepsilon, \varepsilon>0$.

$2^{\circ}$. If $F(z) \in \mathcal{A}_{k}^{2}$, then, as $v \rightarrow k$ (or $v \rightarrow-k$ ), the functions $F(u+i v)$ converge to some limit function in the sense of the $L^{2}(\mathbb{R})$-norm, as well as in the sense of pointwise convergence almost everywhere. This limit function will be denoted by $F(u+i k)$ (respectively, $F(u-i k))$.

$3^{\circ}$. The Fourier transformation is an isomorphism (i.e., a linear, bijective, and twoway continuous map) of the Banach space $M_{k}^{2}$ onto the Banach space $\mathcal{A}_{k}^{2}$.

$4^{\circ}$. The subspace $\mathcal{A}_{*}^{2}$ is dense in the Banach space $\mathcal{A}_{k}^{2}$ for any $k>0$.

$5^{\circ}$. The norm $\mathfrak{n}_{k}$ in $\mathcal{A}_{k}^{2}$ is equivalent to the norm

$$
\widetilde{\mathfrak{n}}_{k}(F):=\left(\int_{\mathbb{R}}\left(|F(u+i k)|^{2}+|F(u-i k)|^{2}\right) d u\right)^{1 / 2} .
$$

Proof. $1^{\circ}$. See [10, $\$ 5.2$, Lemma to Theorem 93].

$2^{\circ}$. See [10, Theorem 97].

$3^{\circ}$. Statement $2^{\circ}$ shows that, in the definition (3.8) of the norm $\mathfrak{n}_{k}$ in the space $\mathcal{A}_{k}^{2}$, we can replace the strict inequality $|v|<k$ with the nonstrict one $|v| \leq k$; i.e., the norm can be defined by formula (3.1). Next, we can argue as in Lemma 2.1.

$4^{\circ}$. Since the Fourier transformation takes the spaces $M_{*}^{2}$ and $M_{k}^{2}$ onto the respective spaces $\mathcal{A}_{*}^{2}$ and $\mathcal{A}_{k}^{2}$ isomorphically, it suffices to show that $M_{*}^{2}$ is dense in $M_{k}^{2}$. Let $C_{c}(\mathbb{R})$ 
denote the set of all continuous and compactly supported functions on $\mathbb{R}$. Then $C_{c}(\mathbb{R})$ is dense in $M_{k}^{2}$, and we see that $M_{*}^{2}$ is dense in $M_{k}^{2}$ because $C_{c}(\mathbb{R}) \subset M_{*}^{2}$.

$5^{\circ}$. We use the notation employed in the proof of Lemma 3.1. Let $F \in \mathcal{A}_{k}^{2}$, and let $f_{v}(x)$ be the inverse Fourier transform of the function $F(u+i v)$ of the variable $u$. By [10, Theorem 97], for $|v| \leq k$ we have formula (3.4), where $f(x)=f_{0}(x)$. Using (3.4) and the Parseval formula for the Fourier transformation, we get (3.2), whence we deduce the inequality

$$
\begin{aligned}
\int_{\mathbb{R}}|F(u+i v)|^{2} d u & \leq 2 \pi \int_{\mathbb{R}}|f(x)|^{2}\left(e^{2 k x}+e^{-2 k x}\right) d x \\
& =\int_{\mathbb{R}}\left(|F(u+i k)|^{2}+|F(u-i k)|^{2}\right) d u .
\end{aligned}
$$

This implies that $\mathfrak{n}_{k}^{2}(F) \leq \widetilde{\mathfrak{n}}_{k}^{2}(F)$, which shows that the two norms are equivalent, because $\tilde{\mathfrak{n}}_{k}^{2}(F) \leq 2 \mathfrak{n}_{k}^{2}(F)$.

Lemma 3.6. Suppose that a function $F(z), z=u+i v$, is analytic and bounded, and has no zeros in the strip $|v|<\pi / 4$. Then for any smaller strip $|v| \leq l<\pi / 4$ there exists $A=A(F, l)>0$ such that

$$
\frac{1}{|F(z)|} \leq \exp (A \cosh 2 u), \quad z=u+i v,|v| \leq l .
$$

Proof. There is no loss of generality in assuming that $F(0)=1$. The map $\alpha: z \mapsto w=$ $\tan (i z)$ is a bijective conformal mapping of the strip $\Pi_{\pi / 4}$ onto the unit disk $D$. Then the function $G(w)=F\left(\alpha^{-1}(w)\right)$ is bounded and analytic in $D$, and $G(w)$ has no zeros in $D, G(0)=1$. It is known (see [12, Chapter I, Theorem 9]) that the above conditions imply the following estimate:

$$
|G(w)| \geq M^{-\frac{2|w|}{1-|w|}}
$$

where $M=\sup _{w \in D}|G(w)|$. Since

$$
\frac{2|w|}{1-|w|}=\frac{2|w|(1+|w|)}{1-|w|^{2}} \leq \frac{4}{1-|w|^{2}}, \quad w \in D
$$

from (3.18) we obtain

$$
|G(w)| \geq M^{-\frac{4}{1-|w|^{2}}} .
$$

For $w=\tan (i z), z=u+i v$, inequality (3.13) yields

$$
1-|w|^{2} \geq \frac{1}{2} e^{-2|u|} \cos 2 v \geq \frac{1}{4}(\cosh 2 u)^{-1} \cos 2 v .
$$

Passing in (3.19) to the variable $z$ and using (3.20), we get

$$
|F(z)| \geq M^{-16 \cosh 2 u(\cos 2 v)^{-1}} .
$$

Since $|v| \leq l<\pi / 4,(3.21)$ implies (3.17) with $A=\frac{16}{\cos 2 l} \ln M$.

Corollary 3.2. Suppose that a function $F(z), z=u+i v$, is analytic and bounded, and has no zeros in the strip $|v|<k$. Then for any strip $|v| \leq l<k$ there exists $A=A(F, k, l)>0$ such that

$$
\frac{1}{|F(z)|} \leq \exp \left(A \cosh \frac{\pi u}{2 k}\right), \quad z=u+i v,|v| \leq l .
$$


By a Blaschke factor in the strip $\Pi_{\pi / 4}$ we shall mean the function

$$
\chi_{\lambda}(z)=\frac{\overline{\tan (i \lambda)}}{|\tan (i \lambda)|} \frac{\tan (i \lambda)-\tan (i z)}{1-\overline{\tan (i \lambda)} \tan (i z)},
$$

where $\lambda \in \Pi_{\pi / 4}, \lambda \neq 0$. For $\lambda=0$, the Blaschke factor has the form

$$
\chi_{0}(z)=\tan (i z) \text {. }
$$

If $\Lambda=\left\{\lambda_{n}=u_{n}+i v_{n}\right\}$ is a finite or countable collection of numbers in the strip $\Pi_{\pi / 4}$ (each $\lambda_{n} \in \Lambda$ is counted with a finite multiplicity), then the function

$$
B(z)=\prod_{n} \chi_{\lambda_{n}}(z)
$$

is called the Blaschke product in the strip $\Pi_{\pi / 4}$. We denote by $B_{m}(z)$ the product of $m$ first Blaschke factors.

After the change $\gamma=\tan (i \lambda)$ and $w=\tan (i z)$, the functions (3.23) and (3.24) turn into the usual Blaschke factors in the unit disk $D$, and the product $B(z)$ turns into the usual Blaschke product $\widetilde{B}(w)$ in $D$ constructed for the points $\gamma_{n}=\tan \left(i \lambda_{n}\right)$.

It is well known (see, e.g., [11]) that such a Blaschke product $\widetilde{B}(w)$ converges in $D$ if and only if the Blaschke condition (3.10) is satisfied. Under this condition, $|\widetilde{D}(w)|<1$ is analytic in $D,|\widetilde{B}(w)|<1$ in $D$, and the zeros of $\widetilde{B}(w)$ coincide with the points $\gamma_{n}$ (with the corresponding multiplicities). Since condition (3.10) with $\gamma_{n}=\tan \left(i \lambda_{n}\right)$ is equivalent to (3.9) (see the proof of Lemma 3.4), we see that the Blaschke product (3.25) in the strip $\Pi_{\pi / 4}$ converges if and only if condition (3.9) is fulfilled, and then $B(z)$ is analytic in $\Pi_{\pi / 4},|B(z)|<1$, and the zeros of $B(z)$ coincide with the points of $\Lambda$ (with the corresponding multiplicities). We also note that on the boundary of the strip $\Pi_{\pi / 4}$ the module of each Blaschke factor is equal to 1, i.e., $\left|\chi_{\lambda}(z)\right|=1$ if $|v|=\pi / 4$, and $\left|\chi_{\lambda}(z)\right| \rightarrow 1$ as $u \rightarrow \infty$ uniformly in $v$.

In the strip $\Pi_{k}, k>0$, by the Blaschke factors we mean

$$
\chi_{k, \lambda}(z):=\chi_{\frac{\pi \lambda}{4 k}}\left(\frac{\pi z}{4 k}\right), \quad \lambda \in \Pi_{k},
$$

and the corresponding Blaschke product is the function $B(z)$ defined by formula (3.25) with $\chi_{\lambda_{n}}(z)$ replaced by $\chi_{k, \lambda_{n}}(z)$.

Lemma 3.7. Let $F(z) \in \mathcal{A}_{k}^{2}$, and let $\Lambda=\left\{\lambda_{n}=u_{n}+i v_{n}\right\}$ be a finite or countable set of (not necessarily all) zeros of $F$ (each $\lambda_{n} \in \Lambda$ is counted with some finite multiplicity not exceeding the multiplicity of $\lambda$ as a zero of $F$ ). Then:

1) the Blaschke product $B(z)$ converges in the strip $\Pi_{k}$, and the function $F(z) / B(z)$ belongs to $\mathcal{A}_{k}^{2}$;

2 ) if the set $\Lambda$ is countable, then, for any $0<l<k$, as $m \rightarrow \infty$ we have

$$
F(z) / B_{m}(z) \rightarrow F(z) / B(z)
$$

in the space $\mathcal{A}_{l}^{2}$, where $B_{m}(z)$ is the product of the first $m$ Blaschke factors of the product $B(z)$.

Proof. 1) We prove the lemma for the strip $\Pi_{\pi / 4}$; the general case of $\Pi_{k}$ reduces to this by the transformation $\Pi_{\pi / 4} z \mapsto \pi z /(4 k)$.

If $\Lambda$ consists of zeros of $F \in \mathcal{A}_{\pi / 4}^{2}$, then condition (3.9) is fulfilled (this follows from Lemma 3.4 and Remark 3.1). Therefore, the Blaschke product $B(z)$ converges in the strip $\Pi_{\pi / 4}$. Since the set of zeros of $B(z)$ coincides with $\Lambda$, the function $\Psi(z):=F(z)(B(z))^{-1}$ is analytic in $\Pi_{\pi / 4}$.

Let $B_{m}(z)$ be the product of the first $m$ Blaschke factors in the product $B(z)$. We check that the function $\Psi_{m}(z):=F(z)\left(B_{m}(z)\right)^{-1}$ belongs to $\mathcal{A}_{\pi / 4}^{2}$. 
Let $P$ be an arbitrary compact subset of $\Pi_{\pi / 4}$ such that the points $\lambda_{1}, \lambda_{2}, \ldots, \lambda_{m}$ lie in the interior of $P$, and let $d=\sup \left\{\left|z_{1}-z_{2}\right|: z_{1}, z_{2} \in P\right\}$ be the diameter of $P$. On the set $P$ the function $\left|\Psi_{m}(z)\right|$ is bounded from above, and outside of $P$ so is the function $\left|B_{m}(z)\right|^{-1}$. Put

$$
c_{1}=\sup \left\{\left|\Psi_{m}(z)\right|: z \in P\right\}, \quad c_{2}=\sup \left\{\left|B_{m}(z)\right|^{-1}: z \in \Pi_{\pi / 4} \backslash P\right\} .
$$

If $|v|<\pi / 4$, we can write

$$
\int_{\mathbb{R}}\left|\Psi_{m}(u+i v)\right|^{2} d u \leq c_{1}^{2} d+c_{2}^{2} \int_{\mathbb{R}}|F(u+i v)|^{2} d u \leq c_{1}^{2} d+c_{2}^{2} \mathfrak{n}_{\pi / 4}^{2}(F) .
$$

Since the right-hand side in (3.26) is independent of $v$, we have $\Psi_{m} \in \mathcal{A}_{\pi / 4}^{2}$.

Since $\left|B_{m}(z)\right|=1$ for $|v|=\pi / 4$, we have $\widetilde{\mathfrak{n}}_{\pi / 4}\left(\Psi_{m}\right)=\widetilde{\mathfrak{n}}_{\pi / 4}(F)\left(\widetilde{\mathfrak{n}}_{\pi / 4}\right.$ is the norm defined in (3.15)). Therefore, using statement $5^{\circ}$ of Lemma 3.5, we obtain the estimate

$$
\mathfrak{n}_{\pi / 4}\left(\Psi_{m}\right) \leq C \mathfrak{n}_{\pi / 4}(F),
$$

with some constant $C$ (the proof of Lemma 3.5 shows that $C=\sqrt{2}$ ), i.e.,

$$
\int_{\mathbb{R}}\left|\Psi_{m}(u+i v)\right|^{2} d u \leq C^{2} \mathfrak{n}_{\pi / 4}^{2}(F) .
$$

For each fixed $v$, the sequence $\left|\Psi_{m}(u+i v)\right|^{2}$ is formed by nonnegative functions of the variable $u$ that are measurable on $\mathbb{R}$, and as $m \rightarrow \infty$ these functions converge to $\left|\Psi_{m}(u+i v)\right|^{2}$ pointwise almost everywhere on $\mathbb{R}$. Then, by the Fatou theorem (see [13), the function $|\Psi(u+i v)|^{2}$ is integrable and satisfies

$$
\int_{\mathbb{R}}|\Psi(u+i v)|^{2} d u \leq C^{2} \mathfrak{n}_{\pi / 4}^{2}(F),
$$

whence $\Psi \in \mathcal{A}_{\pi / 4}^{2}$.

2) We fix an arbitrary $v$ such that $|v|<\pi / 4$. Then $\left|\Psi(u+i v)-\Psi_{m}(u+i v)\right|^{2} \rightarrow 0$ as $m \rightarrow \infty$ for a.e. $u \in \mathbb{R}$ (except for $u$ such that $u+i v \in \Lambda$ ). Since $\left|\Psi_{m}(z)\right| \leq|\Psi(z)|$ for $z \in \Pi_{\pi / 4}$, we have

$$
\left|\Psi(u+i v)-\Psi_{m}(u+i v)\right|^{2} \leq 2\left(|\Psi(u+i v)|^{2}+\left|\Psi_{m}(u+i v)\right|^{2}\right) \leq 4|\Psi(u+i v)|^{2} .
$$

The Lebesgue dominated convergence theorem shows that

$$
\int_{\mathbb{R}}\left|\Psi(u+i v)-\Psi_{m}(u+i v)\right|^{2} d u \rightarrow 0
$$

as $m \rightarrow \infty$. Taking $v= \pm l$ and using statement $5^{\circ}$ of Lemma 3.5, we see that $\tilde{\mathfrak{n}}_{l}\left(\Psi-\Psi_{m}\right) \rightarrow 0$, so that $\Psi_{m} \rightarrow \Psi$ in the space $\mathcal{A}_{l}^{2}$ for $0<l<\pi / 4$.

Lemma 3.8. Let $F(z) \in \mathcal{A}_{k / 3}^{2}, k>0$, and let

$$
\Phi_{\varepsilon}(z):=\exp \left(-\varepsilon \cosh \frac{\pi z}{k}\right), \quad \varepsilon>0 .
$$

Then $F(z) \Phi_{\varepsilon}(z) \in \mathcal{A}_{k / 3}^{2}$ and $F(z) \Phi_{\varepsilon}(z) \rightarrow F(z)$ as $\varepsilon \rightarrow 0$ in the space $\mathcal{A}_{k / 3}^{2}$.

Proof. It is easily seen that

$$
\left|\Phi_{\varepsilon}(z)\right|=\exp \left(-\varepsilon \cosh \frac{\pi u}{k} \cos \frac{\pi v}{k}\right),
$$

where $z=u+i v$. Since $\cos \frac{\pi v}{k} \geq \frac{1}{2}$ if $|v| \leq k / 3$, we have

$$
\left|\Phi_{\varepsilon}(z)\right| \leq \exp \left(-\frac{\varepsilon}{2} \cosh \frac{\pi u}{k}\right) \leq 1
$$


whence $F(z) \Phi_{\varepsilon}(z) \in \mathcal{A}_{k / 3}^{2}$. Since $F(z) \Phi_{\varepsilon}(z)-F(z) \rightarrow 0$ as $\varepsilon \rightarrow 0$ at every point of the strip $|v| \leq k / 3$ and $\left|F(z) \Phi_{\varepsilon}(z)-F(z)\right| \leq 2|F(z)|$, the Lebesgue dominated convergence theorem shows that

$$
\int_{\mathbb{R}}\left|F(u+i v) \Phi_{\varepsilon}(u+i v)-F(u+i v)\right|^{2} d u \rightarrow 0 \text { as } \varepsilon \rightarrow 0
$$

for each $v$ with $|v| \leq k / 3$. In particular, taking $v= \pm k / 3$, we see that

$$
\widetilde{\mathfrak{n}}_{k / 3}\left(F \Phi_{\varepsilon}-F\right) \rightarrow 0 \text { as } \varepsilon \rightarrow 0,
$$

where $\widetilde{\mathfrak{n}}_{k}$ is the norm defined in (3.15). Since this norm is equivalent to $\widetilde{\mathfrak{n}}_{k}$, we conclude that $F \Phi_{\varepsilon} \rightarrow F$ in the Banach space $\mathcal{A}_{k / 3}^{2}$.

Let $I$ be a nontrivial closed ideal in the algebra $\mathcal{A}_{*}^{2}$. For any $k>0$, let $I_{k}$ denote the closure of $I$ in the space $\mathcal{A}_{k}^{2}$. The definition of the topology in $\mathcal{A}_{*}^{2}$ shows that

$$
I=\bigcap_{k>0} I_{k}
$$

Lemma 3.9. Let $F(z) \in I_{k}$, let $F(z)$ be bounded in the strip $\Pi_{k}$, and let $G$ be an arbitrary function analytic and bounded in $\Pi_{k}$. Then $F(z) G(z) \in I_{k}$.

Proof. 1) First we treat the case where $G(z) \in \mathcal{A}_{*}^{2}$. By the definition of $I_{k}$, there is a sequence of functions $F_{m} \in I$ such that $F_{m} \rightarrow F$ in $\mathcal{A}_{k}^{2}$ as $m \rightarrow \infty$. Since $G(z)$ is bounded in $\Pi_{k}$ (see statement $1^{\circ}$ of Lemma 3.5 ), we have $F_{m} G \rightarrow F G$ in the space $\mathcal{A}_{k}^{2}$, and we see that $F G \in I$ because $F_{m} G \in I_{k}$.

2) Next, suppose that $G(z) \in \mathcal{A}_{k}^{2}$ and $G(z)$ is bounded in $\Pi_{k}$. Since $\mathcal{A}_{*}^{2}$ is dense in $\mathcal{A}_{k}^{2}$ (statement $4^{\circ}$ of Lemma 3.5), there is a sequence $G_{m} \in \mathcal{A}_{*}^{2}$ such that $G_{m} \rightarrow G$ as $m \rightarrow \infty$ in the space $\mathcal{A}_{k}^{2}$. Since $F$ is bounded in $\Pi_{k}$, it follows that $F G_{m} \rightarrow F G$ in $\mathcal{A}_{k}^{2}$, and since $F G_{m} \in I_{k}$ and $I_{k}$ is closed in $\mathcal{A}_{k}^{2}$, we conclude that $F G \in I_{k}$.

3) Now we pass to the general case: $G(z)$ is an arbitrary analytic function bounded in the strip $\Pi_{k}$.

Consider the function $F(z) G(z) \Phi_{\varepsilon}(z / 3)$, where $\Phi_{\varepsilon}(z)$ is as defined in Lemma 3.8. Estimate (3.11) shows that the function $G(z) \Phi_{\varepsilon}(z / 3)$ belongs to $\mathcal{A}_{k}^{2}$ and is bounded in $\Pi_{k}$. By item 2) above, we have $F(z) G(z) \Phi_{\varepsilon}(z / 3) \in I_{k}$. On the other hand, since $F(z) G(z) \in \mathcal{A}_{k}^{2}$, we can use Lemma 3.8 to show that $F(z) G(z) \Phi_{\varepsilon}(z / 3) \rightarrow F(z) G(z)$ as $\varepsilon \rightarrow 0$ in the space $\mathcal{A}_{k}^{2}$. But the subspace $I_{k}$ is closed in $\mathcal{A}_{k}^{2}$, so that $F(z) G(z) \in I_{k}$.

Let $\Lambda$ be the set of all zeros of the ideal $I$, and let $r_{\lambda}$ be the multiplicity of $\lambda \in \Lambda$. We denote by $\Lambda_{k}$ the subset of $\Lambda$ that lies in the strip $\Pi_{k}$.

Lemma 3.10. Let $F(z) \in I_{k}$, let $F$ be bounded in the strip $\Pi_{k}$, let $\lambda \in \Pi_{k}$ be a zero of $F$, and let $s$ be the multiplicity of $\lambda$. If $\lambda \notin \Lambda_{k}$, or $\lambda \in \Lambda_{k}$ and $s>r_{\lambda}$, then the function $F(z)\left(\chi_{k, \lambda}(z)\right)^{-1}$ belongs to $I_{k}$.

Proof. Without loss of generality, we may assume that $k=\pi / 4$. The definition of the set $\Lambda_{\pi / 4}$ shows that there exists $G(z) \in I$ such that either $\lambda$ is not a zero of $G$, or it is a zero of $G$ of multiplicity $r<s$. In the first case, we agree that $r=0$.

Let

$$
\Theta(z)=F(z)\left(\chi_{\lambda}(z)\right)^{-r}, \quad \Theta_{0}(z)=G(z)\left(\chi_{\lambda}(z)\right)^{-r} .
$$

Since $\Theta_{0}(\lambda) \neq 0$, we may assume that $\Theta_{0}(\lambda)=1$. The functions

$$
\widetilde{\Theta}(z):=\Theta(z)\left(\chi_{\lambda}(z)\right)^{-1} \text { and } \widetilde{\Theta}_{0}:=\left(\Theta_{0}(z)-1\right)\left(\chi_{\lambda}(z)\right)^{-1}
$$

are analytic in $\Pi_{\pi / 4}$. Then we have

$$
-\widetilde{\Theta}_{0} \Theta+\widetilde{\Theta} \Theta_{0}=\Theta\left(\chi_{\lambda}\right)^{-1} .
$$


Multiplying by $\left(\chi_{\lambda}\right)^{r}$, we get

$$
-\widetilde{\Theta}_{0} F+\widetilde{\Theta} G=F\left(\chi_{\lambda}\right)^{-1} .
$$

The functions $F(z)$ and $G(z)$ are bounded on the strip $\Pi_{\pi / 4}$ (for $G$ this follows from statement $1^{\circ}$ of Lemma 3.5). Therefore, the functions $\widetilde{\Theta}(z)$ and $\widetilde{\Theta}_{0}(z)$ are also bounded on $\Pi_{\pi / 4}$. Lemma 3.9 implies that $\widetilde{\Theta}_{0} F$ and $\widetilde{\Theta} G$ belong to $I_{\pi / 4}$. Now, to show that $F\left(\chi_{\lambda}\right)^{-1} \in I_{\pi / 4}$, it remains to refer to (3.33).

Lemma 3.11. For any $k>0$, in the subspace $I_{k}$ we can find a function $\Psi(z)$ such that $\Psi(z)$ is bounded in $\Pi_{k}$ and the set of zeros of $\Psi$ in $\Pi_{k}$ coincides with the set $\Lambda_{k}$.

Proof. We fix an arbitrary number $K>k$. Let $F(z)$ be a nonzero function in the ideal $I$, and let $\mathfrak{N}_{F, k}$ denote the set of all zeros (with multiplicity) of $F$ that lie in $\Pi_{k}$. The multiplicity of $\lambda \in \mathfrak{N}_{F, k}$ will be denoted by $m_{\lambda}$.

Among the zeros of $F$ in $\Pi_{k}$, there are some belonging to $\Lambda_{k}$ (we call them "necessary zeros"), but there can be some "extra zeros." Let $\widetilde{\Lambda}_{k}$ denote the set of all extra zeros of $F$ in the strip $\Pi_{k}$, i.e., $\lambda \in \widetilde{\Lambda}_{k}$ if $\lambda \in \mathfrak{N}_{F, k}$ and either $\lambda \notin \Lambda_{k}$, or $\lambda \in \Lambda_{k}$ but $m_{\lambda}>r_{\lambda}$ ( $r_{\lambda}$ is the multiplicity of $\lambda$ in the collection $\Lambda_{k}$ ). Assume that each $\lambda \in \widetilde{\Lambda}_{k}$ belongs to $s_{\lambda}$ with multiplicity $s_{\lambda}$, where

$$
s_{\lambda}= \begin{cases}m_{\lambda} & \text { if } \lambda \notin \Lambda_{k}, \\ m_{\lambda}-r_{\lambda} & \text { if } \lambda \in \Lambda_{k} .\end{cases}
$$

We denote by $B(z)$ the product of all Blaschke factors $\chi_{\lambda, k}(z)$ in $\Pi_{k}$ constructed for the collection $\widetilde{\Lambda}_{k}$, and let $B_{m}(z)$ be the product of the first $m$ factors of $B(z)$.

As $\Psi(z)$ we take $F(z)(B(z))^{-1}$. We have $F(z) \in I \subset I_{K}$, and $F(z)$ is bounded in $\Pi_{K}$. By Lemma 3.10, $F(z)\left(B_{m}(z)\right)^{-1} \in I_{K} \subset I_{k}$. By statement $2^{\circ}$ of Lemma 3.7, $F\left(B_{m}\right)^{-1} \rightarrow F B^{-1}$ as $m \rightarrow \infty$ in the space $\mathcal{A}_{l}^{2}$ for any $l$ with $k<l<K$. Consequently, $\Psi(z)=F(z)(B(z))^{-1} \in I_{l} \subset I_{k}$. The function $\Psi(z)$ has only necessary zeros in $\Pi_{k}$ and is bounded on that strip.

Proof of Theorem 3.1. $1^{\circ}$. Let $I$ be a nontrivial closed ideal in the algebra $\mathcal{A}_{*}^{2}$; we prove that $I$ is localizable.

Let $\Lambda$ be the set of zeros of $I, r_{\lambda}$ the multiplicity of a zero $\lambda \in \Lambda, \Lambda_{k}$ the subsets of zeros lying in $\Pi_{k}$. Suppose that $F(z) \in \mathcal{A}_{*}^{2}$ and that each $\lambda \in \Lambda$ is a zero of $F$ of multiplicity at least $r_{\lambda}$. We need to check that $F \in I$. Relation (3.36) shows that it suffices to prove that $F \in I_{k / 3}$ for any $k>0$.

Let $\Phi_{\varepsilon}(z)$ be as in Lemma 3.8; then $F(z) \Phi_{\varepsilon}(z) \in \mathcal{A}_{k / 3}^{2}$ and $F(z) \Phi_{\varepsilon}(z) \rightarrow F(z)$, as $\varepsilon \rightarrow 0$, in the space $\mathcal{A}_{k / 3}^{2}$. By Lemma 3.11, there is a function $\Psi \in I_{k}$ such that the set of zeros of $\Psi$ in $\Pi_{k}$ coincides with $\Lambda_{k}$. Let $B(z)$ be the product of all Blaschke factors $\chi_{\lambda, k}(z)$ over all $\lambda \in \Lambda_{k}$. Then, by Lemma 3.7, the functions $F(z) / B(z)$ and $\Psi(z) / B(z)$ belong to $\mathcal{A}_{k}^{2}$. Since $\Psi(z) / B(z)$ has no zeros in $\Pi_{k}$, from Corollary 3.2 it follows that in the strip $\Pi_{k / 3}$ we have

$$
\left|\frac{B(z)}{\Psi(z)}\right| \leq \exp \left(A \cosh \frac{\pi u}{2 k}\right)
$$

where $A>0$ is some number, $z=u+i v$. Combining this with (3.31), we see that

$$
\left|\frac{B(z)}{\Psi(z)} \Phi_{\varepsilon}(z)\right| \leq \exp \left(-\frac{\varepsilon}{2} \cosh \frac{\pi u}{k}+A \cosh \frac{\pi u}{2 k}\right)
$$

for $|v| \leq k / 3$. Since $\cosh \frac{\pi u}{k}$ grows faster than $\cosh \frac{\pi u}{2 k}$ as $u \rightarrow \infty$, inequality (3.35) shows that the function $B(z)(\Psi(z))^{-1} \Phi_{\varepsilon}(z)$ belongs to $\mathcal{A}_{k / 3}^{2}$ and is bounded on $\Pi_{k / 3}$. Since the 
function $F(z) / B(z)$ belongs to $\mathcal{A}_{k}^{2}$, it is bounded on $\Pi_{k / 3}$; therefore,

$$
\frac{F(z)}{\Psi(z)} \Phi_{\varepsilon}(z)=\frac{F(z)}{B(z)} \frac{B(z)}{\Psi(z)} \Phi_{\varepsilon}(z)
$$

belongs to $\mathcal{A}_{k / 3}^{2}$ and is bounded on $\Pi_{k / 3}$.

We can represent $F \Phi_{\varepsilon}$ in the form $F \Phi_{\varepsilon}=\left(F \Psi^{-1} \Phi_{\varepsilon}\right) \Psi$. Since $F \Psi^{-1} \Phi_{\varepsilon} \in \mathcal{A}_{k / 3}^{2}$, $\Psi \in I_{k} \subset I_{k / 3}$, and these functions are bounded on $\Pi_{k / 3}$, it follows that $F \Phi_{\varepsilon} \in I_{k / 3}$ by Lemma 3.9. Since the subset $I_{k / 3}$ is closed in $\mathcal{A}_{k / 3}^{2}$ and $F \Phi_{\varepsilon} \rightarrow F$ as $\varepsilon \rightarrow 0$ in the space $\mathcal{A}_{k / 3}^{2}$ (see Lemma 3.8), we have $F \in I_{k / 3}$, Thus, the ideal $I$ is localizable.

$2^{\circ}$. Let $\Lambda$ be the set of zeros of a nontrivial closed ideal $I \subset \mathcal{A}_{*}^{2}$. We prove that conditions (i) and (ii) of Theorem 1.1 are fulfilled.

Since the ideal $I$ is nonzero, condition (i) is satisfied. Let $\Lambda_{k}=\left\{\lambda_{n}=u_{n}+i v_{n}\right\}$ be the set of all zeros of the ideal $I$ that lie in the strip $\Pi_{k}, k>0$ (each zero is counted with its multiplicity). Let $F(z)$ be an arbitrary nonzero function belonging to $I$. For any $K>0$, the function $F$ is a nonzero bounded function of class $\mathcal{A}_{K}^{2}$. If $K>k$, then the points of $\Lambda_{k}$ are included in $\Pi_{K}$; therefore, by Remark 3.1, the collection $\Lambda_{k}$ satisfies the condition

$$
\sum_{n} e^{-\frac{\pi}{2 K}\left|u_{n}\right|} \cos \frac{\pi}{2 K} v_{n}<\infty
$$

Since $\left|v_{n}\right|<k$, we have

and (3.36) implies that

$$
\cos \frac{\pi}{2 K} v_{n}>\cos \frac{\pi k}{2 K}>0
$$

$$
\sum_{n} e^{-\frac{\pi}{2 K}\left|u_{n}\right|}<\infty
$$

We put $\varepsilon=\pi /(2 K)$; since $K$ can be as large as we wish, $\varepsilon>0$ is as small as we wish. Then condition (3.37) takes the form

$$
\sum_{n} e^{-\varepsilon\left|u_{n}\right|}<\infty \text { for all } \varepsilon>0 .
$$

Assume that the $\lambda_{n}$ in $\Lambda_{k}$ are enumerated in the order of increasing of $\left|u_{n}\right|$ :

$$
\left|u_{1}\right| \leq\left|u_{2}\right| \leq\left|u_{3}\right| \leq \cdots .
$$

We prove that (3.38) implies the relation

$$
\frac{\left|u_{n}\right|}{\ln n} \rightarrow \infty \text { as } n \rightarrow \infty
$$

(of course, provided the collection $\Lambda_{k}$ is infinite). Suppose the contrary; then there is a number $c>0$ and a subsequence $u_{n_{j}}, j=1,2,3, \ldots$, such that $\left|u_{n_{j}}\right| / \ln n_{j} \leq c$, whence

$$
S_{n_{j}}=\sum_{n=1}^{n_{j}} e^{-\varepsilon\left|u_{n}\right|} \geq n_{j} e^{-\varepsilon\left|u_{n_{j}}\right|} \geq n_{j}^{1-\varepsilon c} .
$$

Taking $\varepsilon=(2 c)^{-1}$, we see that $S_{n_{j}} \geq n_{j}^{1 / 2}$, i.e., $S_{n_{j}} \rightarrow \infty$ as $j \rightarrow \infty$, which contradicts (3.38). Consequently, condition (3.40) is fulfilled and, with it, condition (ii) is also fulfilled.

$3^{\circ}$. We pass to the proof of the "if" part in statement $2^{\circ}$ of Theorem 1.1. Let $\Lambda$ be an arbitrary collection of complex numbers satisfying conditions (i) and (ii) of Theorem 1.1. We must construct a closed ideal $I \subset \mathcal{A}_{*}^{2}$ whose set of zeros coincides with $\Lambda$. We show that, for this, it suffices to construct a nonzero function $F \in \mathcal{A}_{*}^{2}$ such that every point 
$\lambda \in \Lambda$ is a zero of $F$ of multiplicity at least $r_{\lambda}$ ( $r_{\lambda}$ is the multiplicity of $\lambda$ in $\Lambda$ ), but $F$ may have other zeros.

Indeed, suppose $F$ has such properties. Let $N_{F}$ denote the set of zeros of $F$. Suppose $\lambda \in N_{F}$ and $r$ is the multiplicity of the zero $\lambda$. If $\lambda \notin \Lambda$, we put

$$
F_{\lambda}(z):=F(z)(z-\lambda)^{-r}
$$

and if $\lambda \in \Lambda$, we put

$$
F_{\lambda}(z):=F(z)(z-\lambda)^{-\left(r-r_{\lambda}\right)} .
$$

It is easily seen that $F_{\lambda}(z) \in \mathcal{A}_{*}^{2}$. Let $I$ be the closed ideal in $\mathcal{A}_{*}^{2}$ generated by all functions $F_{\lambda}(z), \lambda \in N_{F}$. The zeros of the ideal $I$ are only points of $\Lambda$, and every $\lambda \in \Lambda$ is a zero of multiplicity $r_{\lambda}$.

$4^{\circ}$. Let $\Lambda$ be an arbitrary collection of complex numbers satisfying (i) and (ii) of Theorem 1.1, let $r_{\lambda}$ be the multiplicity of $\lambda$ in $\Lambda$, and let $\Lambda_{k}$ be the part of $\Lambda$ that lies in the strip $\Pi_{k}, k>0$.

We construct a sequence of functions $\Psi_{m}(z), m=1,2,3, \ldots$, with the following properties:

1) $\Psi_{m} \in \mathcal{A}_{m+1}^{2}$

2) each $\lambda \in \Lambda_{m+1}$ is a zero of $\Psi_{m}$ of multiplicity at least $r_{\lambda}$;

3) $\mathfrak{n}_{m / 3}\left(\Psi_{m+1}-\Psi_{m}\right)<2^{-m-1}$

4) $\mathfrak{n}_{1 / 3}\left(\Psi_{1}\right)=1$.

For the role of $\Psi_{1}(z)$ we take any function of class $\mathcal{A}_{2}^{2}$ whose set of zeros coincides with $\Lambda_{2}$ and whose $\mathfrak{n}_{1 / 3}$-norm is equal to 1 (such a function exists in accordance with Corollary 3.7; we use the fact that (3.14) follows from (ii)). Suppose the functions $\Psi_{1}$, $\Psi_{2}, \ldots, \Psi_{m}$ have already been constructed; we build $\Psi_{m+1}$.

Let $\Psi$ be an arbitrary function in $\mathcal{A}_{m+2}^{2}$ whose set of zeros is $\Lambda_{m+2}$. We denote by $\alpha(z)$ the product of all Blaschke factors $\chi_{m+1, \lambda}(z)$ in $\Pi_{m+1}$ constructed for the points of $\Lambda_{m+1}$. Let $G(z)=\Psi(z) / \alpha(z)$. Then $G(z) \in \mathcal{A}_{m+1}^{2}$ (by Lemma 3.7), and $G(z)$ has no zeros in $\Pi_{m+1}$. By Corollary 3.2, in the strip $\Pi_{m}$, the function $(G(z))^{-1}=\alpha(z) / \Psi(z)$ admits the estimate

$$
\left|\frac{\alpha(z)}{\Psi(z)}\right| \leq \exp \left(A \cosh \frac{\pi u}{2 m}\right), \quad z=u+i v
$$

where $A>0$ is a constant independent of $z$.

Let $\Phi_{\varepsilon}(z)$ be the function in (3.30) with $k=m$. In the strip $\Pi_{m / 3}$ we consider the function $\Psi_{m}(z) \Phi_{\varepsilon}(z)$, choosing $\varepsilon$ so as to ensure the inequality

$$
\mathfrak{n}_{m / 3}\left(\Psi_{m}-\Psi_{m} \Phi_{\varepsilon}\right)<2^{-m-2}
$$

(this is possible by Lemma 3.8). The function $\Psi_{m} / \alpha$ belongs to $\mathcal{A}_{m+1}^{2}$ (by Lemma 3.7) and, consequently, is bounded on $\Pi_{m / 3}$. Estimates (3.41) and (3.31) show that on $\Pi_{m / 3}$ we have

$$
\left|\frac{\alpha(z)}{\Psi(z)} \Phi_{\varepsilon}(z)\right| \leq \exp \left(-\frac{\varepsilon}{2} \cosh \frac{\pi u}{m}+A \cosh \frac{\pi u}{2 m}\right) .
$$

Now, from (3.43) we see that $\alpha(z)(\Psi(z))^{-1} \Phi_{\varepsilon}(z) \in \mathcal{A}_{m / 3}^{2}$, whence

$$
\Psi_{m} \Psi^{-1} \Phi_{\varepsilon}=\left(\Psi_{m} \alpha^{-1}\right)\left(\alpha \Psi^{-1} \Phi_{\varepsilon}\right) \in \mathcal{A}_{m / 3}^{2} .
$$

By statement $4^{\circ}$ of Lemma 3.5 , the set $\mathcal{A}_{*}^{2}$ is dense in $\mathcal{A}_{m / 3}^{2}$, so that there exists a function $H(z) \in \mathcal{A}_{*}^{2}$ such that

$$
\mathfrak{n}_{m / 3}\left(\frac{\Psi_{m}}{\Psi} \Phi_{\varepsilon}-H\right)<\frac{2^{m-2}}{M},
$$


where

$$
M=\max \left\{|\Psi(z)|: z \in \Pi_{m / 3}\right\} .
$$

Put $\Psi_{m+1}(z)=H(z) \Psi(z)$. Since $\Psi \in \mathcal{A}_{m+2}^{2}$ and $H$ is bounded in $\Pi_{m+2}$, we have $\Psi_{m+1} \in \mathcal{A}_{m+2}^{2}$. Any point $\lambda \in \Lambda_{m+2}$ is a zero of $\Psi$ of multiplicity $r_{\lambda}$, and hence it is a zero of $\Psi_{m+1}$ of multiplicity at least $r_{\lambda}$. Using (3.45), we get

$$
\mathfrak{n}_{m / 3}\left(\Psi_{m} \Phi_{\varepsilon}-\Psi_{m+1}\right)=\mathfrak{n}_{m / 3}\left(\left(\frac{\Psi_{m}}{\Psi} \Phi_{\varepsilon}-H\right) \Psi\right) \leq M \frac{2^{-m-2}}{M}=2^{-m-2},
$$

which implies, with the help of (3.42), that

$$
\mathfrak{n}_{m / 3}\left(\Psi_{m+1}-\Psi_{m}\right) \leq \mathfrak{n}_{m / 3}\left(\Psi_{m+1}-\Psi_{m} \Phi_{\varepsilon}\right)+\mathfrak{n}_{m / 3}\left(\Psi_{m} \Phi_{\varepsilon}-\Psi_{m}\right) \leq 2^{-m-1},
$$

proving property 3$)$.

$5^{\circ}$. As was shown in item $3^{\circ}$ above, now it remains to construct a nonzero function $F \in \mathcal{A}_{*}^{2}$ such that each $\lambda \in \Lambda$ is a zero of $F$ of multiplicity at least $r_{\lambda}$.

Let $\Psi_{m}$ be a sequence of functions that satisfy conditions 1)-4) of item $4^{\circ}$ above. We check that for any $z \in \mathbb{C}$ the sequence of complex numbers $\Psi_{m}(z)$ converges, and if $F(z)=\lim _{m \rightarrow \infty} \Psi_{m}(z)$, then the function $F(z)$ is as required.

Indeed, in each strip $\Pi_{k}$, the functions $\Psi_{m}(z)$ are well defined for $m>k-1$, and they constitute a Cauchy sequence in the norm $\mathfrak{n}_{k}$ for $m>3 k$ (see property 3 )). Consequently, the sequence $\Psi_{m}$ converges in the norm $\mathfrak{n}_{k}$, which implies uniform convergence on the compact subsets of $\mathfrak{n}_{k}$, and hence, pointwise convergence. Denoting by $F(z)$ the limit function, we see that $F \in \mathcal{A}_{k}^{2}$ for any $k>0$, so that $F \in \mathcal{A}_{*}^{2}$. By property 2), all points of $\Lambda_{k}$ are zeros of $\Psi_{m}$ for $m>k-1$; therefore, all points of $\Lambda$ are zeros of $F$. Finally, the function $F(z)$ is nonzero, because in the space $\mathcal{A}_{1 / 3}^{2}$ we have

$$
F=\Psi_{1}+\sum_{m=1}^{\infty}\left(\Psi_{m+1}-\Psi_{m}\right)
$$

and properties 2) and 3) imply that

$$
\mathfrak{n}_{1 / 3}(F) \geq \mathfrak{n}_{1 / 3}\left(\Psi_{1}\right)-\sum_{m=1}^{\infty} \mathfrak{n}_{1 / 3}\left(\Psi_{m+1}-\Psi_{m}\right) \geq 1-\sum_{m=1}^{\infty} 2^{-m-1}=\frac{1}{2} .
$$

It should be noted that the basic ideas of the proof of Theorem 3.1 are taken from Rashevskiiı's paper [7, where a close problem was considered; namely, all closed quasiideals were described in the topological vector space $\mathfrak{F}$ formed by all odd functions of class $\mathcal{A}_{*}^{2}$.

\section{§4. Spectral Synthesis in OTHER FUnCtion SPACES}

In this section we present two other classes of function spaces with the spectral synthesis property. We employ the notation of $\S 1$; in particular, $\mathcal{E}$ is the space of indefinitely differential functions on $\mathbb{R}$, and $\mathcal{D}^{\prime}$ is the space of distributions on $\mathbb{R}$.

Let $\mathcal{F}$ be a translation invariant space. We shall say that $\mathcal{F}$ is a function space of free growth if

$$
\mathcal{E} \subseteq \mathcal{F} \subseteq \mathcal{D}^{\prime},
$$

with continuous inclusions. As examples of such spaces, we mention the spaces $C^{d}(\mathbb{R})$ of $d$ times differentiable functions on $\mathbb{R}\left(d \in \mathbb{Z}_{+} \cup\{\infty\}\right)$, and also the spaces $L_{\text {loc }}^{p}(\mathbb{R})$ of all measurable functions $f(x)$ on $\mathbb{R}$ for which the seminorms

$$
|f|_{K, p}:=\left(\int_{K}|f(x)|^{p} d x\right)^{1 / p}
$$

are finite; here $K$ is an arbitrary compact set in $\mathbb{R}$, and $1 \leq p<\infty$. 
Theorem 4.1. Any function space $\mathcal{F}$ of free growth admits spectral synthesis. The spectra of all possible nontrivial invariant subspaces $H \subset \mathcal{F}$ coincide with the finite or countable subsets $\Lambda \subset \mathbb{C}$ (each $\lambda \in \Lambda$ is counted with some finite multiplicity $r_{\lambda}$ ) such that the following condition is satisfied:

(i) there exists a nonzero entire function $\Psi(z)$ such that each $\lambda \in \Lambda$ is a zero of $\Psi$ of multiplicity at least $r_{\lambda}$, and

$$
|\Psi(z)| \leq A e^{B|\operatorname{Im} z|}(1+|z|)^{C}
$$

for some constants $A, B, C$ independent of $z$ (such functions are the Fourier transforms of distributions with compact support).

Proof. Let $\mathcal{F}$ be a function space of free growth. Arguing as in the proof of Theorem 2.1, we check that the invariant subspaces in $\mathcal{F}$ are in one-to-one correspondence with those in $\mathcal{E}$ : with every invariant subspace $H \subset \mathcal{F}$ we can associate $H_{0}=H \cap \mathcal{E} \subset \mathcal{E}$. The following well-known fact should be used: if $f \in \mathcal{D}^{\prime}$ and $\varphi \in \mathcal{D}$, then $f * \varphi \in \mathcal{E}$ and, for $\varphi$ fixed, the map $f \mapsto f * \varphi$ from $\mathcal{D}^{\prime}$ to $\mathcal{E}$ is continuous (see, e.g., 9]).

Next, we argue as in Theorem 2.2 to show that any function space $\mathcal{F}$ of free growth has the spectral synthesis property if the space $\mathcal{E}$ has this property, and that the descriptions of all possible spectra of the invariant subspaces in $\mathcal{F}$ and $\mathcal{E}$ coincide. For the space $\mathcal{E}$, the spectral synthesis property, as well as the description of the spectra of invariant subspaces, follows from the results of Schwartz's paper [2.

Now we define yet another class of function spaces. For any $\nu \in \mathbb{R}$ and $m \in \mathbb{Z}_{+}$, we denote by $\mathfrak{C}_{(\nu)}^{(m)}$ the Banach space of all $m$ times differentiable functions $f(x)$ on $\mathbb{R}$ such that the norm

$$
|f|_{(\nu)}^{(m)}:=\sup \left\{\left(1+x^{2}\right)^{\nu / 2}\left|\partial^{\alpha} f(x)\right|: x \in \mathbb{R}, 0 \leq \alpha \leq m\right\}
$$

is finite. For these spaces we have the continuous embeddings

$$
\mathfrak{C}_{(\nu)}^{(m)} \subseteq \mathfrak{C}_{\left(\nu^{\prime}\right)}^{\left(m^{\prime}\right)}, \quad m \geq m^{\prime}, \nu \geq \nu^{\prime} .
$$

The space

$$
\mathfrak{C}_{(\nu)}^{(\infty)}:=\bigcap_{m \in \mathbb{Z}_{+}} \mathfrak{C}_{(\nu)}^{(m)}
$$

is a complete locally convex space with the topology given by the system of norms $\left\{|\cdot|_{(\nu)}^{(m)}, \quad m \in \mathbb{Z}_{+}\right\}$. Obviously,

$$
\mathfrak{C}_{(\nu)}^{(\infty)} \subseteq \mathfrak{C}_{\left(\nu^{\prime}\right)}^{(\infty)}, \quad \nu \geq \nu^{\prime}
$$

and the inclusions are continuous. We introduce the spaces

$$
\mathcal{S}:=\bigcap_{\nu \in \mathbb{R}} \mathfrak{C}_{(\nu)}^{(\infty)}, \quad \mathcal{O}:=\bigcup_{\nu \in \mathbb{R}} \mathfrak{C}_{(\nu)}^{(\infty)} .
$$

The spaces $\mathcal{S}$ and $\mathcal{O}$ are complete locally convex spaces with the topologies of the projective and inductive limits of the topological vector spaces $\mathfrak{C}_{(\nu)}^{(\infty)}$. The space $\mathcal{S}$ coincides with the usual Schwartz space of infinitely differentiable functions whose derivatives of any order decay faster than any power of $|x|^{-1}$ as $x \rightarrow \infty$. The space $\mathcal{O}$ consists of all infinitely differentiable functions whose derivatives can grow as $x \rightarrow \infty$ no faster than some power of $|x|$. Various properties of the spaces $\mathcal{S}$ and $\mathcal{O}$ can be found, e.g., in [6]. The space $\mathcal{S}^{\prime}$ dual to $\mathcal{S}$ consists of all distributions of slow growth. Equipped with the weak topology $\sigma\left(\mathcal{S}^{\prime}, \mathcal{S}\right)$, the space $\mathcal{S}^{\prime}$ is a complete locally convex space.

We say that a translation invariant space $\mathcal{F}$ is a function space of polynomial growth if

$$
\mathcal{O} \subseteq \mathcal{F} \subseteq \mathcal{S}^{\prime}
$$


with continuous embeddings. As examples of such spaces, we mention $\mathcal{O}, \mathcal{S}^{\prime}$, and the spaces

$$
\mathfrak{C}_{*}^{(m)}:=\bigcup_{\nu \in \mathbb{R}} \mathfrak{C}_{(\nu)}^{(m)}, \quad m \in \mathbb{Z}_{+},
$$

with the topology of the inductive limit of the Banach spaces $\mathfrak{C}_{(\nu)}^{(m)}$.

Theorem 4.2. 1) Any function space $\mathcal{F}$ of polynomial growth admits spectral synthesis. The spectra $\Lambda$ of all possible invariant subspaces $H \subseteq \mathcal{F}$ are real, and the multiplicities $r_{\lambda}$ of points $\lambda \in \Lambda$ can be infinite.

2) A subset $\Lambda \subseteq \mathbb{R}$ (each $\lambda \in \Lambda$ is counted with some multiplicity $\left.r_{\lambda} \in \mathbb{N} \cup\{\infty\}\right)$ is the spectrum of some invariant subspace $H \subseteq \mathcal{F}$ if and only if the following conditions are satisfied:

(i) the set $\Lambda_{\infty}:=\left\{\lambda \in \Lambda: r_{\lambda}=\infty\right\}$ is closed in $\mathbb{R}$;

(ii) the set $\Lambda_{\text {fin }}:=\Lambda \backslash \Lambda_{\infty}$ is at most countable, and all limit points of this set (if they exist) belong to $\Lambda_{\infty}$.

Proof. 1) Let $\mathcal{F}$ be an arbitrary function space of polynomial growth. Arguing as in the proof of Theorem 2.1, we check that there is a one-to-one correspondence between the collections of invariant subspaces in the spaces $\mathcal{F}$ and $\mathcal{S}^{\prime}$ : with an invariant subspace $H \subset \mathcal{F}$ we can associate the invariant subspace $[H] \subset \mathcal{S}^{\prime}\left([H]\right.$ is the closure of $H$ in $\left.\mathcal{S}^{\prime}\right)$. The following well-known property should be used: if $f \in \mathcal{S}^{\prime}$ and $\varphi \in \mathcal{D}$ (or even $\varphi \in \mathcal{S}^{\prime}$ ), then $f * \varphi \in \mathcal{O}$ (see, e.g., [6, $\S 3.2]$ ).

Next we argue as in Theorem 2.2 to show that an arbitrary function space $\mathcal{F}$ of polynomial growth has the spectral synthesis property if the space $\mathcal{S}^{\prime}$ has this property, and that the descriptions of all possible invariant subspaces in $\mathcal{F}$ and in $\mathcal{S}^{\prime}$ coincide. Therefore, it suffices to prove Theorem 4.1 for the space $\mathcal{S}^{\prime}$.

2) The spectral synthesis property for $\mathcal{S}^{\prime}$ was proved in the paper [3] by Schwartz. For any invariant subspace $H \subset \mathcal{S}^{\prime}$, let $H^{\perp}$ be its orthogonal complement in $\mathcal{S}$, i.e.,

$$
H^{\perp}=\{\varphi \in \mathcal{S}:\langle f, \varphi\rangle=0 \forall f \in \mathcal{S}\},
$$

and let $I=\Phi\left(H^{\perp}\right)$ be the Fourier image of $H^{\perp}$. The map $H \mapsto I$ establishes a one-toone correspondence between the invariant subspaces in $\mathcal{S}^{\prime}$ and the closed ideals in the algebra $\mathcal{S}$ (with the pointwise product of functions).

Arguing as in the proof of Lemma 2.3, we check that an invariant subspace $H \subset \mathcal{S}^{\prime}$ admits spectral synthesis if and only if the ideal $I$ corresponding to $H$ is localizable, and then the spectrum $\Lambda$ coincides with the set of zeros of the ideal $I$ (with multiplicity).

In the paper [14 by Molchanov, it was proved that conditions (i) and (ii) of Theorem 4.2 are necessary and sufficient for a subset $\Lambda \subseteq \mathbb{R}$ to coincide with the set of zeros of some closed ideal in $\mathcal{S}$. This completes the proof of Theorem 4.2.

\section{REFERENCES}

[1] N. Bourbaki, Espaces vectoriels topologiques. Chapters 1-5, new edition, Masson, Paris, 1981. MR0633754 (83k:46003)

[2] L. Schwartz, Théorie générale des fonctions moyenne-périodiques, Ann. of Math. (2) 48 (1947), 857-929. MR0023948 (9:428c)

[3] _ Analyse et synthèse harmoniques dans les espaces de distributions, Canadian J. Math. 3 (1951), 503-512. MR0044754 (13:473c)

[4] N. K. Nikol'skiı̌, Invariant subspaces in operator theory and function theory, Itogi Nauki i Tekhniki Mat. Anal., vol. 12, VINITI, Moscow, 1974, pp. 199-412; English transl. in J. Soviet Math. 5 (1976), no. 1, 2. MR0430821 (55:3826)

[5] L. Székelyhidi, Discrete spectral synthesis and its applications, Springer, Berlin, 2006. MR2279454 (2009d:43001) 
[6] L. R. Volevich and S. G. Gindikin, Generalized functions and convolution equations, Nauka, Moscow, 1994. (Russian) MR1379334 (97g:46041)

[7] P. K. Rashevskiı̌, Description of closed invariant subspaces in certain function spaces, Trudy Moskov. Mat. Obshch. 38 (1979), 139-185; English transl. in Trans. Moscow Math. Soc. 1980, no. 2. MR0544938 (81d:43007)

[8] J. E. Gilbert, On the ideal structure of some algebras of analytic functions, Pacific J. Math. 35 (1970), no. 3, 625-634. MR0412439 (54:565)

[9] G. E. Shilov, Mathematical analysis: Second special course, Nauka, Moscow, 1965. (Russian) MR0219869 (36:2943)

[10] E. Titchmarsh, Introduction to the theory of Fourier integrals, Gostekhizdat, Moscow, 1948; English transl., Chelsea Publ. Co., New York, 1986. MR.0942661 (89c:42002)

[11] J. Garnett, Bounded analytic functions, Pure Appl. Math., vol. 96, Acad. Press, New York-London, 1981. MR0628971 (83g:30037)

[12] B. Ya. Levin, Distributions of zeros of entire functions, Gostekhizdat, Moscow, 1956; English transl., Amer. Math. Soc., Providence, RI, 1964. MR0087740(19:402c) MR0156975 (28:217)

[13] A. N. Kolmogorov and S. V. Fomin, Elements of the theory of functions and functional analysis, Nauka, Moscow, 1976; English transl. from 1st ed., Vol. 1, Graylock Press, Rochester, NY, 1957; Vol. 2, 1961. MR0435771(55:8728) MR0085462(19:44d) MR0118796 (22:9566a)

[14] V. F. Molchanov, Elementary representations of the Laguerre group, Mat. Zametki 23 (1978), no. 1, 31-39; English transl., Math. Notes 23 (1978), no. 1-2, 19-23. MR0507244 (58:22402)

Petrozavodsk State University, Lenin Ave. 33, Petrozavodsk 185910, Russia

E-mail address: platonov@su.karelia.ru

Received 14/JUN/2009

Translated by A. PLOTKIN 